\title{
Cohesive traction-separation relations for plate tearing under mixed mode loading
}

Andersen, R. G.; Woelke, P. B.; Nielsen, K. L.

Published in:

European Journal of Mechanics A - Solids

Link to article, DOI:

10.1016/j.euromechsol.2018.03.016

Publication date:

2018

Document Version

Peer reviewed version

Link back to DTU Orbit

Citation (APA):

Andersen, R. G., Woelke, P. B., \& Nielsen, K. L. (2018). Cohesive traction-separation relations for plate tearing under mixed mode loading. European Journal of Mechanics A - Solids, 71, 199-209.

https://doi.org/10.1016/j.euromechsol.2018.03.016

\section{General rights}

Copyright and moral rights for the publications made accessible in the public portal are retained by the authors and/or other copyright owners and it is a condition of accessing publications that users recognise and abide by the legal requirements associated with these rights.

- Users may download and print one copy of any publication from the public portal for the purpose of private study or research.

- You may not further distribute the material or use it for any profit-making activity or commercial gain

- You may freely distribute the URL identifying the publication in the public portal

If you believe that this document breaches copyright please contact us providing details, and we will remove access to the work immediately and investigate your claim 


\title{
Cohesive Traction-Separation Relations for Plate Tearing under Mixed Mode Loading
}

\author{
R.G. Andersen ${ }^{\mathrm{a}, *}$, P.B. Woelke ${ }^{\mathrm{b}}$, K.L. Nielsen ${ }^{\mathrm{a}}$ \\ ${ }^{a}$ Department of Mechanical Engineering, Solid Mechanics, Technical University of Denmark, \\ DK-2800 Kgs. Lyngby, Denmark \\ ${ }^{b}$ Thornton Tomasetti, Weidlinger Applied Science Practice, NY 10005-1304, United States
}

\section{Abstract}

The present study investigates a sequence of failure events related to steady-state tearing of large-scale ductile plates by employing the micro-mechanics based GursonTvergaard-Needleman (GTN) model. The fracture process in front of an advancing crack is approximated by a series of 2D plane strain finite element models to facilitate a comprehensive study of mixed mode fracture behavior as well as a parameter study of the cohesive energy and tractions involved in the process. The results from the conducted GTN model simulations are used to define cohesive zone models suitable for plate tearing simulations at large scale. It is found that mixed mode loading conditions can have a significant effect on the cohesive energy as well as relative displacement (in reference to pure mode I loading), while peak traction is practically unaffected. Specifically, increasing mode II contribution leads to monotonic increase of the cohesive energy. In contrast, the effect of mode III is more complicated as it leads to reduction of the mixed mode cohesive energy (in reference to pure mode I loading) at low to medium levels of mode mixity ratios (0-0.3). However, increasing mode III contribution beyond the mode mixity ratio of 0.3 , reverses this trend with cohesive energy potentially

\footnotetext{
*Tel: +45 4525-4020

URL: rgra@mek.dtu.dk (R.G. Andersen)
} 
exceeding the pure mode I level when at mode mixity ratio of 0.6 or higher. This behavior cannot be captured by the interactive cohesive zone models that rely on a simple rotational sweep of mode I traction-separation relation. Depending on the shear mode contribution, i.e., mode II or mode III, these models can lead to overly conservative (mode II) or unconservative (mode III) prediction of the crack growth resistance.

Keywords: Ductile Failure, Crack Growth, Plate Structures, Cohesive Zone Modeling

\section{Introduction}

The main focus of the present work is on determination of the cohesive zone model parameters that can be used to approximate the complex ductile fracture process evolving in large-scale plate tearing under mixed mode loading conditions. When the tearing crack in a large-scale plate has advanced several plate thicknesses, under monotonic loading, and the failure process ahead of the crack tip has reached a steady-state propagation, the energy dissipation proceeds through a sequence of events which includes: i) local thinning that takes place some distance ahead of the crack tip; ii) shear localization that subsequently develops on a smaller scale inside the thinning region closer to the tip, and; iii) final separation that advances the crack (see also discussion in Nielsen and Gundlach, 2017; Nielsen and Hutchinson, 2017). This complex plate tearing process is driven by the mechanism of void nucleation and growth to coalescence and it can be captured by the micro-mechanics based Gurson-Tvergaard-Needleman material model in a full 3D framework (Felter and Nielsen, 2017). To accurately represent the complexity of the plate tearing process, a through-thickness resolution that scales with the dominant void spacing (e.g. $\sim 100 \mu \mathrm{m}$ ) is required (see also Xue et al., 2010; 
Nielsen and Hutchinson, 2012). Such resolution is presently only possible for coupon specimens and small components. Thus, engineers rely heavily on the phenomenological alternatives, such as cohesive zone models embedded in shell elements, to ensure computation times that are short enough for industrial applications (see also discussion in Li and Siegmund, 2002; Woelke et al., 2017).

When modeling failure in thin-walled structures using shell elements, one needs to consider constraints related to the plane stress condition, which is an inherent assumption in shell mechanics. Maintaining a plane stress state within a shell element requires that its in-plane dimensions are larger than the thickness. Since the height of the localized neck is on the order of sheet thickness, only a single element can be used to represent necking and failure. This is, of course, not sufficient to capture the detailed geometry of local thinning. To address this deficiency, cohesive zone models can be employed to represent the effects that cannot be captured by large shell elements. In this case, the cohesive zone must take over as soon as through-thickness localization starts and describe the remaining part of the fracture process. Tvergaard and Hutchinson (1992) studied the relation between ductile crack propagation and the cohesive zone parameters that govern the fracture process. An interesting conclusion in their work is that in plane strain, it is the peak traction and the cohesive energy that primarily control the tearing response, whereas the shape of the traction-separation relation is of minor importance. In a later work, Nielsen and Hutchinson (2012) made an attempt to design a cohesive traction-separation relation for extensive crack propagation in tough ductile plates where the tearing energy and the peak traction were direct outcomes of the underlying micro-mechanics. Here, by considering a cross-section of the plate with 
the normal along the crack growth direction, modeled in 2D plane strain, a detailed micro-mechanical study of the slant failure was performed using the shear extended GTN modeling framework (Gurson, 1977; Tvergaard, 1990; Nahshon and Hutchinson, 2008). In this approach, the cohesive zone takes over once the peak traction of the plate cross-section has been reached and both the localization process and final failure were treated in a rigorous, but phenomenological, manner. Thus, the cohesive zone model reflects the actual micro-mechanics that lead to crack propagation once the fracture process has settled into a steady-state (Scheider and Brocks, 2006). Despite only treating mode I loading, the traction-separation relation proposed by Nielsen and Hutchinson (2012) has been successfully applied by Woelke et al. $(2013,2015)$ to investigate large-scale plate tearing. By adopting the micro-mechanics based tractionseparation relation, a near perfect match to experimentally measured load-deflection curves was obtained for the macroscopic structural response. As an aside, Woelke et al. (2015) concluded that for plane stress conditions, the shape of the traction-separation relation is also important for accurate prediction of crack growth resistance. However, these considerations were limited to pure mode I loading, whereas real life structures often encounter mixed mode loading. A common practice in modeling mixed mode loading with cohesive zone relies on essentially a rotational sweep of the normal mode I traction-separation relation, $T\left(\delta_{n}\right)$, into the tangential separation (between fracture surfaces) space such that the traction curves become $T\left(\delta_{t 1}\right)$ and $T\left(\delta_{t 2}\right)$ in pure mode II or pure mode III, respectively (see Eq. (1)). The work of separation is thus traditionally assumed to be unchanged between modes (Li and Siegmund, 2002) and mode 
mixity is calculated as

$$
\Gamma_{0}=\int_{0}^{\lambda_{0}} T(\lambda) d \lambda, \text { with } \lambda=\sqrt{\left(\frac{\delta_{n}}{\delta}\right)^{2}+\left(\frac{\delta_{t 1}}{\delta}\right)^{2}+\left(\frac{\delta_{t 2}}{\delta}\right)^{2}}
$$

Here, $\Gamma_{0}$ is the work of separation (equal for all modes), $\delta_{n}$ is the normal separation, $\delta_{t 1}$ and $\delta_{t 2}$ are the tangential separations of the fracture surfaces related to mode II and mode III separation, respectively. The present study will show that this approach does not represent reality in ductile plate tearing under mixed mode loading. It will be demonstrated that after peak traction is reached, the work of separation depends on mode mixity. The goal of the current study is twofold: i) to highlight the effects of mode mixity on the overall cohesive energy as well as other parameters defining the tractionseparation relation, and; ii) to develop a new mixed mode traction-separation relation that readily fits into the framework of combining plane stress shell elements with cohesive zone modeling without sacrificing the accuracy for mixed mode loading. Details of the traction-separation relations will be developed through micro-mechanics modeling, which in turn will form the basis for guidelines on how parameterized tractionseparation relations can be constructed without compromising accuracy. The employed modeling framework has been adopted from Nielsen and Hutchinson (2012), but with modifications to take out-of-plane actions into account.

The paper outlines the constitutive relations and finite element model in Section 2. The problem formulation is described in Section 3, after which the cohesive zone model is defined in Section 4 by identifying key parameters to be extracted from the micro-mechanics based numerical simulations. Results are given in Section 5 with focus on improving accuracy within the field of cohesive zone modeling of large-scale plate tearing. Conclusions are listed in Section 6. 


\section{Model: constitutive relations and finite element formulation}

\subsection{Material description}

The undamaged (matrix) material in this study is assumed to follow a true stresslogarithmic strain power hardening relation described as:

$$
\varepsilon= \begin{cases}\frac{\sigma_{M}}{E} & , \text { for } \sigma_{M}<\sigma_{y} \\ \frac{\sigma_{y}}{E}\left(\frac{\sigma_{M}}{\sigma_{y}}\right)^{1 / N} & , \text { for } \sigma_{M} \geq \sigma_{y}\end{cases}
$$

where $\sigma_{y}$ is the initial yield stress, $E$ is the Young's modulus, and $N$ is the hardening exponent. To account for the softening effect of the damage that evolves during severe plastic straining, the material is assumed to be governed by void growth to coalescence and to follow the flow rule for a porous ductile GTN material (Gurson, 1977) with the yield surface modified by Tvergaard (1981)

$$
\Phi=\frac{\sigma_{e}^{2}}{\sigma_{M}^{2}}+2 q_{1} f^{*} \cosh \left(\frac{q_{2}}{2} \frac{\sigma_{k}^{k}}{\sigma_{M}}\right)-\left(1+\left(q_{1} f^{*}\right)^{2}\right)=0 .
$$

Here, $\sigma_{e}=\sqrt{3 s_{i j} s^{i j} / 2}$ is the effective macroscopic Mises stress, with $s^{i j}=\sigma^{i j}-$ $G^{i j} G_{k l} \sigma^{k l} / 3$ being the stress deviator where $G_{i j}$ and $G^{i j}$ are the co- and contravariant component of the metric tensor, respectively, associated with the deformed geometry. The microscopic stress in the matrix material is denoted $\sigma_{M}$, whereas $q_{1}$ and $q_{2}$ are fitting parameters introduced by Tvergaard (1981), and $f^{*}$ is a function of the porosity that takes void coalescence into account. Tvergaard and Needleman (1984) suggested the following phenomenological model to accelerate the damage increase once microvoids link up in the coalescence process:

$$
f^{*}= \begin{cases}f & , \text { for } f \leq f_{C} \\ f_{C}+\frac{\bar{f}_{U}-f_{C}}{f_{F}-f_{C}}\left(f-f_{C}\right) & , \text { for } f>f_{C}\end{cases}
$$


where $f$ is the accumulated damage (or porosity), $f_{C}$ and $f_{F}$ are the critical and final porosity, respectively. The ultimate damage, $\bar{f}_{U}$, is defined as $1 / q_{1}$.

The development of damage in the material is partly controlled by void growth and partly a shear contribution, such that the total rate of damage reads:

$$
\dot{f}=\dot{f}_{\text {growth }}+\dot{f}_{\text {shear }}
$$

where a damage contribution from nucleating voids is omitted for clarity of results in the present study. Void growth follows from plastic incompressibility and can be expressed as:

$$
\dot{f}_{\text {growth }}=(1-f) G^{i j} \dot{\eta}_{i j}^{p}
$$

where $\dot{\eta}_{i j}^{p}$ is the increment of the plastic strain tensor. It is known, however, that evolution of the damage predicted by the GTN model stops if the stress triaxiality goes to zero, e.g. for a pure shear loading case. In order to investigate the effect of shear damage, the shear extension introduced by Nahshon and Hutchinson (2008) will be considered as part of the analysis. The governing equation for the shear contribution to total damage is:

$$
\dot{f}_{\text {shear }}=k_{\omega} f \omega(\boldsymbol{\sigma}) \frac{s^{i j} \dot{\eta}_{i j}^{p}}{\sigma_{e}}
$$

where $\omega(\boldsymbol{\sigma})=1-\left(27 J_{3} /\left(2 \sigma_{e}^{3}\right)\right)^{2}$. Here, $J_{3}$ is the third invariant of Cauchy stress deviator and $k_{\omega}$ is the amplification factor for the shear contribution, which typically lies in the range of $[0 ; 3]$ (see also Tvergaard and Nielsen, 2010). It is worth mentioning that the Nahshon-Hutchinson extension is purely phenomenological and it is, therefore, only considered as a supplement to the GTN model in this analysis. When $k_{\omega}=0$, the material model reduces to the GTN model. It should also be stressed, that $k_{\omega}=$ 
0 unless stated otherwise. All material parameters are listed in Table 1 (see also Tvergaard, 1990, for further details).

\subsection{Finite element formulation}

The analyses are carried out in a finite strain setting, which has been embedded in a quasi-static framework using a total Lagrangian formulation. The incremental expansion of the principle of virtual work reads:

$$
\int_{V}\left(\dot{\tau}^{i j} \delta \eta_{i j}+\tau^{i j} \dot{u}_{, i}^{k} \delta u_{k, j}\right) \mathrm{d} V=\int_{S} \dot{T}^{i} \delta u_{i} \mathrm{~d} S
$$

where $\tau^{i j}$ is the Kirchhoff stress tensor, $\eta_{i j}$ is the Lagrangian strain tensor, $u_{i}$ is the displacement field and $T^{i}$ are the tractions. Here, the Lagrangian strain tensor is given as; $\eta_{i j}=\frac{1}{2}\left(u_{i, j}+u_{j, i}+u_{, i}^{k} u_{k, j}\right)$, and the relation between the rate of strains and the rate of Kirchhoff stresses is given by:

$$
\dot{\tau}^{i j}=L^{i j k l} \dot{\eta}_{k l}
$$

where $L^{i j k l}$ is the incremental modulus. The incremental computational procedure follows that of Tvergaard (1990).

\section{Problem formulation}

\subsection{Model description}

The considered boundary value problem is inspired by the work of Nielsen and Hutchinson (2012) who examined a crack propagating at steady-state through a large-scale plate subjected to pure mode I loading. The plate tearing process is illustrated in Fig. 1, where the plate dimensions in the $x_{1}$ - and $x_{2}$-direction are much larger than 
the out-of-plane plate thickness (dimension in the $x_{3}$-direction). In the 2D set-up employed, a section with the normal along the crack growth direction, i.e., the $x_{1}-x_{3}$-plane, is imagined cut from the full 3D plate and the deformation experienced by this section is assumed to be plane strain $\left(\eta_{22}=0\right)$. As the plastic flow localizes, and all subsequent deformation takes place in this region (see Fig. 1C), the domain above and below the thinning region elastically unloads and this enforces a constraint on the deformation along the $x_{2}$-direction leading to a near tip plane strain condition (see also Nielsen and Hutchinson, 2017). The section considered maintains an initial aspect ratio of $L_{0} / W_{0}=3$ throughout, and this ensures that the complete localization process is captured, see Fig. 2. The adopted set-up is an approximation as no gradients can develop in the crack growth direction.

The present study covers both mode I-mode II loading and mode I-mode III loading and thus, two different sets of boundary conditions are employed for the geometric model, see Eqs. (3) and (4). In order to capture the material softening beyond onset of local thinning, prescribed displacements are employed in all the computations. For the mode I-mode II load case, prescribed displacements in the $x_{1}$ - and $x_{2}$-direction are enforced according to (see also Fig. 2(a)):

$$
\begin{array}{r}
u_{1}\left(x_{1}=L_{0} / 2, x_{3} \in\left[-W_{0} / 2, W_{0} / 2\right]\right)=\Delta_{I} \\
u_{1}\left(x_{1}=-L_{0} / 2, x_{3} \in\left[-W_{0} / 2, W_{0} / 2\right]\right)=-\Delta_{I} \\
u_{2}\left(x_{1} \in\left[9 L_{0} / 19, L_{0} / 2\right], x_{3} \in\left[-W_{0} / 2, W_{0} / 2\right]\right)=\Delta_{I I} \\
u_{2}\left(x_{1} \in\left[-9 L_{0} / 19,-L_{0} / 2\right], x_{3} \in\left[-W_{0} / 2, W_{0} / 2\right]\right)=-\Delta_{I I}
\end{array}
$$

For the mode I-mode III load case, prescribed displacements in the $x_{1^{-}}$and $x_{3^{-}}$ 
direction are enforced according to (see also Fig. 2(b)):

$$
\begin{gathered}
u_{1}\left(x_{1}=L_{0} / 2, x_{3} \in\left[-W_{0} / 2, W_{0} / 2\right]\right)=\Delta_{I} \\
u_{1}\left(x_{1}=-L_{0} / 2, x_{3} \in\left[-W_{0} / 2, W_{0} / 2\right]\right)=-\Delta_{I} \\
u_{3}\left(x_{1} \in\left[L_{0} / 4, L_{0} / 2\right], x_{3}=0\right)=\Delta_{I I I} \\
u_{3}\left(x_{1} \in\left[-L_{0} / 4,-L_{0} / 2\right], x_{3}=0\right)=-\Delta_{I I I}
\end{gathered}
$$

The chosen boundary conditions attempt to mimic the deformation inside the plate section that contains a tearing crack, imposed by global displacements of a large-scale plate. As a result, curvature continuity between the plate section that contains the crack and the remainder of the plate structure (not modelled explicitly) is achieved. Thereby, plastic flow localization near the top or bottom strip is avoided.

The amount of shear (mode II or mode III) contributing to the total load is varied to investigate the influence of the mode mixity on the cohesive traction-separation relation. Results will be presented for mode mixity ratios of $\Delta_{I I} / \Delta_{I}$ (mode I-mode II) and $\Delta_{I I I} / \Delta_{I}$ (mode I-mode III) within the range from 0 to 0.6 .

The computations are carried out using a modified isoparametric 8-node plane strain element employing reduced integration ( $2 \times 2$ Gauss points). The element is modified by expanding the nodal degrees of freedom from two degrees of freedom to three degrees of freedom per node. It is worth mentioning, that the shape function structure is unchanged as the element has no extension in the out-of-plane direction. Thus, the shape functions are only defined in 2D, in the original coordinate system. This also means, that the Jacobian matrix is unchanged since the partial derivatives with respect to the element's out-of-plane axis is zero. However, the strain-displacement matrix is changed when introducing the additional degree of freedom as additional terms in 
the displacement gradient vector are needed. This finite element formulation provides the ability to analyze the model displacements along all three axes, and thus enables conducting the mode mixity investigation without the need for full 3D analysis. The plane strain condition is maintained by constraining the strain component along the crack growth direction to zero $\left(\eta_{22}=0\right)$, although the strain will still have a non-zero elastic and an equal and opposite plastic strain contribution.

Figure 3 illustrates the discretized models. A fine mesh is introduced in the middle region as this is where the local thinning and subsequent slant fracture develops. The elements are initially quadratic in shape and have a side length of $L_{e}=W_{0} / 63$ adopted from Nielsen and Hutchinson (2012). In the bottom and top regions a coarser discretization is used as the deformation remains nearly homogeneous during loading. In these regions, the largest elements have a side length of $L_{e}=W_{0} / 7$. While the GTN model solution is inherently mesh dependent, this only affects the cohesive energy related to the shear localization which is a second order contribution to the cohesive energy (see Fig. 4 and discussion in Nielsen and Hutchinson, 2012).

An imperfection zone, controlled by a small drop in the initial yield stress (see Eq. (5)), is embedded in all models in a $45^{\circ}$ band stretching across the center of the plate. The imperfection ensures that only one active shear band will develop, within the fracture process zone, which otherwise may be symmetric (Nielsen and Hutchinson, 2012).

$$
\begin{gathered}
\sigma_{y}^{I Z}=\sigma_{y}\left[1-\frac{\beta}{2}\left(1+\cos \left(\pi \frac{x_{1}+x_{3}}{2 b}\right)\right)\right] \\
\quad \text { for }\left(-x_{3}-b\right) \leq x_{1} \leq\left(-x_{3}+b\right)
\end{gathered}
$$

where $\beta=0.001$ such that the yield stress is $0.1 \%$ lower in the middle of the band, 
while the width of the imperfection zone is controlled by $b$ (chosen to $\left.W_{0} / 5\right)$. The imperfection zone is illustrated in Fig. 2. It is worth to notice that the initial $45^{\circ}$ inclination of the softer band is a choice (with practically no influence on the peak traction and tearing energy), and the single active shear band will form independently. That is, rather than pre-defining the crack path as in the work of Besson et al. (2013), the damage is free to develop.

\section{Outlining the cohesive zone model for ductile plate tearing}

Figure 4 displays a representative traction-separation relation, extracted from the 2D model setup. The onset of local thinning and the point of intensified shear localization are designated as two key deformation stages that identify the cohesive zone model. The peak traction occurs at the onset of local thinning, which in combination with the point of intensified shear localization (here defined as the deformation state where the damage evolution severely intensifies and coalescence is first initiated, $f=f_{C}$ ) determines the associated cohesive energy (this is in line with the key parameters identified by Tvergaard and Hutchinson, 1992).

Since large shell elements cannot capture the details of the plate thinning and shear localization, cohesive zone is used to represent the deformation after peak traction has been reached. Under mode I plane strain tension condition, one can readily derive the peak traction and corresponding displacement for the cohesive zone model using the Considère condition (see also Nielsen and Hutchinson, 2012). The majority of the energy that goes into the ductile fracture process is dissipated during the local thinning (denoted $\Gamma_{I}$ in Fig. 4) and identified to be the area under the traction-separation relation between the peak traction and the intensification of the shear localization. By 
taking the deformation beyond the intensification of the shear localization, damage will intensify in a narrow region, eventually leading to a complete loss of load carrying capacity. The energy going into this last part of the tearing process is minor (denoted $\Gamma_{I I}$ in Fig. 4), and it is the only part that will be influenced by the inherent mesh dependency of the GTN model (see Section 2). Thus, the cohesive energy, computed from the simulated traction-separation relations, is; $\Gamma_{0}=\Gamma_{I}+\Gamma_{I I}$, and constitutes the energy required for complete separation. A simple bi-linear traction-separation relation, described in Appendix A, is uniquely defined by the peak traction, the point where the load carrying capacity diminishes (here corresponding to the intensification of the shear localization), and the cohesive energy, $\Gamma_{0}$ (the area under the tractionseparation relation). Thus, the focus of the micro-mechanical modeling approach is on accurate determination of these key parameters.

Under mixed mode loading, the cohesive traction-separation relation has to take into account all load contributions (also illustrated in Fig. 2). Thus, effective quantities are introduced for each of the two load cases (mode I-mode II and mode I-mode III) such that the displacements and the forces are computed as:

$$
\Delta_{I-I I}=\sqrt{\Delta_{I}^{2}+\Delta_{I I}^{2}} \text { and } F_{I-I I}=\sqrt{F_{I}^{2}+F_{I I}^{2}} \text { for mode I-mode II }
$$

and

$$
\Delta_{I-I I I}=\sqrt{\Delta_{I}^{2}+\Delta_{I I I}^{2}} \text { and } F_{I-I I I}=\sqrt{F_{I}^{2}+F_{I I I}^{2}} \text { for mode I-mode III }
$$

where $\Delta_{I}$ is the prescribed displacement applied, corresponding to a mode I, which results in a force of $F_{I}$ etc. That is, the total cohesive energy is calculated from the 
effective quantities as:

$$
\Gamma_{0}=\int_{\Delta_{\text {peak }}}^{\Delta_{\text {failure }}} F_{I-I I / I-I I I} \mathrm{~d} \Delta
$$

which naturally accounts for changes to the cohesive energy that originates from changes to both the necking zone and the shear band orientation (if any) for the various loading cases. Recall that no restriction is put on the shear band and that it can form with any inclination angle.

\section{Results}

The load-deformation history for the $2 \mathrm{D}$ plate section is evaluated when subjected to mode I-mode II loading, and subsequently to mode I-mode III loading. The part of the simulated load-deflection curve relevant to the cohesive zone modeling of large-scale plate tearing is then extracted and cohesive traction-separation relations are determined based on the procedure laid out in Section 4. The key parameters, i.e., the peak traction, $T_{\text {peak }}$, the point of intensified shear localization, $\delta_{s}$, and the cohesive energy, $\Gamma_{0}$, are determined for a range of mode mixities and three values of initial porosity, i.e., $f_{0}=0.005, f_{0}=0.01$, and $f_{0}=0.02$.

\subsection{Shear related predictions by the GTN model}

Both modes II and III introduce an overall shear component to the loading on the plate section. To address shear damage and softening, the Nahshon-Hutchinson shear extension is used in the analyses (see Eq. (2)). In relation to this, it is also important to point out that the stress triaxility for the results presented throughout is moderate and resembles that of plane strain tension (being $\sigma_{k}^{k} /\left(3 \sigma_{e}\right) \approx 0.6$ ), and that the $\omega(\boldsymbol{\sigma})$ function introduced by Nahshon and Hutchinson (2008) takes values close to one when 
the deformation is well into the plastic regime (see also discussion in Nielsen and Tvergaard, 2010).

Figure 5 presents the cohesive energy as function of the shear amplification parameter, $k_{\omega}$, which scales the shear damage contribution (see Eq. (2)). Results are shown for; i) the pure mode I loading, ii) a mode I-mode II load case with $\Delta_{I I} / \Delta_{I}=0.3$ in Fig. 5a and with $\Delta_{I I} / \Delta_{I}=0.6$ in Fig. 5b, and iii) a mode I-mode III load case with $\Delta_{I I I} / \Delta_{I}=0.3$ in Fig. 5 a and with $\Delta_{I I I} / \Delta_{I}=0.6$ in Fig. 5b. All results are shown for two distinct values of initial porosity, i.e., $f_{0}=0.005$ and $f_{0}=0.02$ (for comparison, the cohesive energy at different mode mixity ratios are depicted in Figs. 8 and 12 for $\left.k_{\omega}=0\right)$. The shear damage contribution has an equally strong influence on all loading configurations considered. This is tied to the fact that the $\omega$-function takes values close to one in all cases. That is, the shear parameter also influences the pure mode I load case and a nearly linear decrease in the cohesive energy is predicted for increasing shear parameter, $k_{\omega}$. Moreover, the slope on all the curves in Fig. 5 is close to equal although the energy levels are different. Taking the pure mode I case as baseline it is found that introducing mode I-mode II mixity leads to an increase in cohesive energy for all considered values of $k_{\omega}$ and mode mixity ratios. In addition, the distance between comparable curves is nearly constant, as shown in both Figs. 5a and 5b. This is an important finding, since it basically shows that adding in-plane shear loading (i.e. mode II) to mode I tensile deformation increases the cohesive energy. This will be further discussed in Section 5.2. The effect of mode I-mode III is equally pronounced for a moderate mode mixity ratio $\left(\Delta_{I I I} / \Delta_{I}=0.3\right)$, except that the cohesive energy is reduced in reference to pure mode I, i.e., the opposite trend to what was found for 
mode I-mode II interaction. However, cohesive energy reduction is completely reversed at high mode mixity ratio $\left(\Delta_{I I I} / \Delta_{I}=0.6\right)$ and the mixed mode energy curve closely follows the pure mode I case. This behavior is related to changes in the amount of the energy dissipated in the cohesive zone, vs. outside of it, as will be further discussed in Section 5.3.

Another important observation related to the numerical aspect of this study is that the $\omega(\boldsymbol{\sigma})$-function is nearly constant in all analyzed cases. This indicates that neglecting the shear damage contribution by setting $k_{\omega}=0$ will not qualitatively change the results.

\subsection{Mode I-mode II}

The peak traction, related to the onset of local thinning (and where the cohesive elements start governing the response), is evaluated for the various ratios of $\Delta_{I I} / \Delta_{I}$ and presented in Fig. 6. The analyses indicate approximately parabolic decrease in the peak traction for all considered values of initial porosity. While the peak traction gradually decreases with increasing levels of mode II contribution, the drop is not significant, i.e., below $4 \%$ in reference to pure mode I for the largest mode II contribution $\left(\Delta_{I I} / \Delta_{I}=\right.$ 0.6). Thus, it is reasonable to consider the peak traction unaffected by the mode II loading in the development of a parametric cohesive zone model for large-scale plate tearing. Moreover, it is evident from Fig. 6 that the negligible effect of the mode II contribution on peak traction holds for a range of porosities encountered in engineering materials.

Another important point on the traction-separation relation is where the shear localization intensifies. This point is defined by a traction, $T_{s}$, and a relative displace- 
ment, $\delta_{s}$, defined as the far boundary displacement that develops during the deformation between the onset of necking (i.e. at the attainment of peak traction) and the intensification of the shear localization. Both the relative displacement and traction are depicted in Fig. 7 as function of the mode mixity ratio (here normalized by the corresponding values for the pure mode I load case). The displacement at intensified shear localization increases significantly with increasing mode mixity ratio $\Delta_{I I} / \Delta_{I}$. Moreover, the effect is found to be reinforced by increasing the initial porosity - particularly for the high mode mixity ratios. That is, the separation that develops within the cohesive zone has its low point for the pure mode I load case while the added mode II increases the separation by $30-45 \%$, depending on the initial porosity, and yields a more ductile overall response prior to the final material separation. In similar fashion, though opposite trend, the traction at intensified shear localization decreases by 10$12 \%$ for increasing mode mixity ratio. This follows the trend for the peak traction, but the decrease is much greater.

The cohesive energy is presented in Fig. 8 as a function of the mode mixity ratio $\Delta_{I I} / \Delta_{I}$, and normalized by the energy level for that of the pure mode I load case. It is found, that the cohesive energy increases with the mode mixity ratio, which is basically caused by a restricted damage evolution. This increase in cohesive energy is consistent with the results shown in Fig. 7. Since the peak traction remains essentially unchanged, and the drop in traction at intensified shear localization is moderate, the change in cohesive energy is tied to the trends observed in the relative displacement $\delta_{s}$. The additional energy needed for the mixed mode I-mode II fracture is expanded by double curvature bending, and dispersion of plastic localization, as the plate section 
complies with the prescribed boundary conditions. The observations hold for all values of the initial porosity although the overall energy level decreases for increasing initial porosity. The additional energy going into a mode I-mode II fracture, when compared to the pure mode I load case, reaches a level of just below $20 \%$ for the lowest value of the initial porosity $\left(f_{0}=0.005\right)$ and $35 \%$ for the highest values of the initial porosity $\left(f_{0}=0.02\right)$, when considering the largest mode mixity ratio $\left(\Delta_{I I} / \Delta_{I}=0.6\right)$. This is a significant difference, which is very consequential for both understanding of mixed mode fracture, but also for development of more accurate interactive cohesive zone models for large-scale plate tearing.

Small sensitivity of the peak traction to mode mixitiy suggests that simple cohesive models based on a commonly applied rotational sweep of the traction-separation relation (see Eq. (1)), would be appropriate (see Fig. 6). However, increasing contributions of mode mixity have significant effects on both cohesive energy and separation at failure, which cannot be captured with simple rotational mode interaction models. The additional energy dissipated through double curvature bending of the plate section during mode I-mode II loading is simply not accounted for in the pure mode I tractionseparation relation. A simple bi-linear traction-separation relation (see Appendix A) fit to the mode I load case and swept into the mode mixity space (dotted lines) is shown in Fig. 9 together with the cohesive relation obtained in this study (bold lines). The latter sweep is found by plotting the traction-separation relations for each simulated load case where the location in the mode mixity space is controlled by the mode mixity ratio, $\Delta_{I I} / \Delta_{I}$. It is evident from Fig. 9 that the two approaches lead to very different cohesive models and that the relations diverge significantly as the mode mixity ratio 
increases.

\subsection{Mode I-mode III}

The mixed mode I-mode III load case is subsequently considered, and the peak traction is first evaluated in Fig. 10 for various ratios of mode mixity $\left(\Delta_{I I I} / \Delta_{I}\right)$. The analyses indicate that the peak traction reduces gradually with increasing mode III contribution, but this reduction is below $1 \%$ and can be neglected.

Variation of the displacement, $\delta_{s}$, and the traction, $T_{s}$, at intensified shear localization are shown in Fig. 11. As previously, the results are normalized with the corresponding values for the pure mode I load case. Compared to the mode I-mode II load case, the effect of increasing mode III contribution is more complicated. The displacement first decreases by $3-8 \%$ to reach a minimum in the interval $\Delta_{I I I} / \Delta_{I} \in[0.25 ; 0.3]$ depending on the initial porosity. In fact, the minimum is attained at larger mode mixity for increasing initial porosity. After reaching a minimum for $\delta_{s}$, further increase of the mode mixity ratio results in an increase of the relative displacement, which eventually becomes larger than that of the pure mode I load case (except for the highest initial porosity considered here). The predicted traction has a similar behaviour; a decrease to a minimum at $\Delta_{I I I} / \Delta_{I} \in[0.2 ; 0.3]$ depending on the value of the initial porosity is observed and followed by an increase that leads to the level for pure mode I loading. The variation in traction at intensified shear localization is, however, much more moderate at a level below $3 \%$.

Figure 12 presents the normalized cohesive energy as a function of the mode mixity ratio $\Delta_{I I I} / \Delta_{I}$. Introducing mode III loading results in a decrease of the cohesive energy for moderate levels of mode mixity ratios $\left(\Delta_{I I I} / \Delta_{I}<0.3\right)$. For higher ratio, the energy 
level increases. In fact, the trend shows that the energy level for high mode mixity ratios eventually will exceed that of the pure mode I load case. The variation in the cohesive energy level is not surprising considering the variation in the traction and the relative displacement at intensified shear localization. It is noted that the cohesive energy is tied directly to these quantities and as the traction levels remain nearly constant, the change in energy level follows the change in relative displacement. This behavior is fundamentally different from that of the mode I-mode II load case, where a steadily increasing trend was seen. This is caused by the shear banding mechanism, which is basically a self-constituting mode III. The mode III displacement is initially constrained (at low mode mixity), but eventually, increasing mode III loading overcomes this initial constraint encouraging transverse shear displacement (at high mode mixity). In other words, at low mode mixity ratios, the two halves of the plate above and below the shear band want to displace in the direction of the prescribed mode III loading, but the pure mode I loading constrains them, thereby maintaining a high energy. This was also shown by Nielsen and Hutchinson (2012), who investigated two different sets of boundary conditions; i) $\Delta_{I I I}=0$, and ii) $\Delta_{I I I}$ unspecified, and with the conclusion that an increased energy level was present for $\Delta_{I I I}=0$. A slight prescribed mode III will release this constraint and lower the energy level. At the other extreme, the self-constituting mode III will be overruled and the parts are forced to displace at large mode mixity ratios, causing the energy level to rise. This mechanism is also evident when the reaction force acting perpendicular to the large-scale plate surface, is investigated. This reaction force is zero at mode mixity ratio that yields the lowest cohesive energy. 
The above analysis provides a fundamental insight into the mixed mode fracture behavior in large ductile plates. In addition, the parametric studies conducted here offer valuable lessons for formulation of accurate, mixed mode cohesive traction-separation relations. The cohesive relation in the mode I-mode III space is depicted in Fig. 13, where the mode I cohesive energy is swept into the mode mixity space (dotted lines) and the simulated curves from this study is depicted in bold lines. A concave trend is observed in the cohesive traction-separation relation, which is related to the decrease in separation when shear localization intensifies for mixed mode loadings. This observation holds for all considered values of the initial porosity. As the loading becomes dominated by the mode III, the cohesive relation again matches the rotational mode I sweep but the trend suggests that a deviation will emerge to the opposite side comparable to the results for the mode I-mode II load case (see Fig. 9).

\section{Concluding remarks}

Fundamental aspects of mixed mode ductile fracture behavior have been investigated, with a specific focus on the key parameters defining the mixed mode cohesive zone model, suitable for large-scale ductile plate tearing simulations. This work can be considered a natural extension of Nielsen and Hutchinson (2012), where only mode I was considered. Two distinct mode mixities have been investigated, i.e., mode I-mode II and mode I-mode III, at different mode mixity ratios and for three typical values of the initial porosity. The study is based on a finite strain, quasi-static finite element Fortran code implemented with a special 2D plane strain element that can account for out-ofplane deformation and which facilitates studying the two sets of loading conditions.

The study has employed the Gurson-Tvergaard-Needleman material model as well as 
the shear extension to this classical model proposed by Nahshon and Hutchinson (2008).

Initial part of the study has been aimed at investigation of the effect the NahshonHutchinson shear extension of the GTN model under mixed mode loading conditions. While the shear extension does have a significant influence on the cohesive energy, this influence is constant for all mode mixity ratios investigated (including pure mode I). This indicates that for all load scenarios, the traditional GTN model is suitable for the analyses defining the relationships between individual modes. However, it is emphasized that the shear extension is necessary for accurate determination of the cohesive energy.

For simplicity, a bi-linear traction-separation relation has been chosen as reference, as it is easily related to characteristics of the traction-separation relations predicted by the micro-mechanical modeling. The key parameters of the cohesive zone model are: i) the onset of local thinning which gives the peak traction, $T_{\text {peak }}$, ii) the point of intensified shear localization, $\delta_{s}$, and iii) the cohesive energy, $\Gamma_{0}$. The numerical analyses have shown that the peak traction is approximately equal for all load cases, but depends on the initial porosity. Only a slight decrease in peak traction (though never above 4\%), was observed when increasing the mode mixity. On the other hand, the relative displacement at the point of intensified shear localization is dependent not only on the load case, but also on the mode mixity ratio. For the mode I-mode II load case, the displacement increases monotonically for an increasing mode mixity ratio, suggesting an increased ductility in reference to pure mode I case. In the case of the mode I-mode III loading, the relative displacement decreases initially at a relatively low mode mixity ratio of 0.3 , and then increases again when mode III contribution grows. It 
should be noted that, since the cohesive traction remains approximately constant until intensification of shear localization, a direct relationship can be established between displacement and cohesive energy for different mode mixities. Even more importantly, the results show that a commonly applied cohesive zone model that relies on a rotational sweep of mode I traction-separation relation, cannot reproduce the observed trends in behavior. Specifically, using the rotational sweep to analyze mode I-mode II loading condition will lead to overestimated crack growth, and the error becomes larger with increasing mode II contribution. On the other hand, crack growth under mode I-mode III conditions at moderate mode mixity ratio will be underestimated by the cohesive zone model relying on the rotational sweep.

These findings clearly show that mixed mode ductile fracture behavior of large-scale plates is quite complicated and caution is warranted when simulating ductile tearing of large-scale plates with cohesive zone models. Some of the most common methodologies employed today to address these problems, may be inadequate, depending on the objective of the analyses.

\section{Acknowledgements}

The work is financially supported partly by the VILLUM FOUNDATION Young Investigator Programme in the project "A New phenomenon Yet to be resolved in ductile PLATE tearing", grant VKR023451 and partly by the Department of Mechanical Engineering at the Technical University of Denmark in the project "Advancing Numerical Analysis of Large Scale Crack Propagation in Plate Structures". 


\section{References}

Besson, J., McCowan, C., Drexles, E., 2013. Modeling flat to slant fracture transition using the computational cell methodology. Eng. Frac. Mech. 104, 80-95.

Felter, C., Nielsen, K., 2017. Assisted crack tip flipping under Mode I thin sheet tearing. European J. Mech. A/Solids 64, 58-68.

Gurson, A., 1977. Continuum theory of ductile rupture by void nucleation and growth - part I: yield criteria and flow rules for porous ductile madia. ASME J. Eng. Mater. Technol. 99, 2-15.

Li, W., Siegmund, T., 2002. An analysis of crack growth in thin-sheet metal via a cohesive zone model. Engineering Fracture Mechanics 69 (18), 2073-2093.

Nahshon, K., Hutchinson, J., 2008. Modification of the Gurson model for shear. Euro. J. Mech. A/Solids 27, 1-17.

Nielsen, K., Hutchinson, J., 2012. Cohesive traction-separation laws for tearing of ductile metal plates. Int. J. Imp. Eng. 48, 15-23.

Nielsen, K., Tvergaard, V., 2010. Ductile shear failure or plug failure of spot welds modelled by modified Gurson model. Eng. Frac. Mech. 77, 1031-1047.

Nielsen, K. L., Gundlach, C., 2017. Crack tip flipping under mode i tearing: Investigated by x-ray tomography. International Journal of Solids and Structures 118-119, $119-127$.

Nielsen, K. L., Hutchinson, J. W., 2017. Steady-state, elastic-plastic growth of slanted 
cracks in symmetrically loaded plates. International Journal of Impact Engineering $108,286-294$.

Scheider, I., Brocks, W., 2006. Cohesive elements for thin-walled structures. Computational Materials Science 37 (1-2), 101-109.

Tvergaard, V., 1981. Influence of voids on shear band instabilities under plane strain conditions. International Journal of Fracture 17 (4), 389-407.

Tvergaard, V., 1990. Material failure by void growth to coalescence. Advances in applied mechanics 27, 83-151.

Tvergaard, V., Hutchinson, J., 1992. The relation between crack growth resistance and fracture process parameters in elastic-plastic solids. J. Mech. Phys. Solids 40, $1377-1397$.

Tvergaard, V., Needleman, A., 1984. Analysis of the cup-cone fracture in a round tensile bar. Acta Metallurgica 32 (1), 157-169.

Tvergaard, V., Nielsen, K., 2010. Relations between a micro-mechanical model and a damage model for ductile failure in shear. J. Mech. Phys. Solids 58, 1243-1252.

Woelke, P., Hiriyur, B., Nahshon, K., Hutchinson, J., 2017. A practical approach to modeling aluminum weld fracture for structural applications. Eng. Frac. Mech. 175, $72-85$.

Woelke, P. B., Shields, M. D., Abboud, N. N., Hutchinson, J. W., 2013. Simulations of ductile fracture in the idealized ship grounding scenario using phenomenological damage and cohesive zone models. Computational Materials Science 80, 79. 
Woelke, P. B., Shields, M. D., Hutchinson, J. W., 2015. Cohesive zone modeling and calibration for Mode I tearing of large ductile plates. Engineering Fracture Mechanics, 293-305.

Xue, Z., Pontin, M. G., Zok, F. W., Hutchinson, J. W., 2010. Calibration procedures for a computational model of ductile fracture. Engineering Fracture Mechanics 77 (3), $492-509$. 


\section{Tables}

Table 1: Material properties.

\begin{tabular}{lll}
\hline Parameters & Notation & Value \\
\hline Young's modulus & $E$ & $210 \mathrm{GPa}$ \\
Poisson's ratio & $\nu$ & 0.3 \\
Yield stress & $\sigma_{y}$ & $630 \mathrm{MPa}$ \\
Hardening exponent & $N$ & 0.1 \\
Initial porosity & $f_{0}$ & {$[0.005 ; 0.01 ; 0.02]$} \\
Critical porosity & $f_{C}$ & 0.15 \\
Final porosity & $f_{F}$ & 0.4 \\
Gurson fitting parameters & $q_{1}, q_{2}$ & $1.5,1.0$ \\
Nahshon-Hutchinson shear parameter & $k_{\omega}$ & {$[0.0 ; 1.0 ; 2.0 ; 3.0]$} \\
\hline
\end{tabular}




\section{Figures}

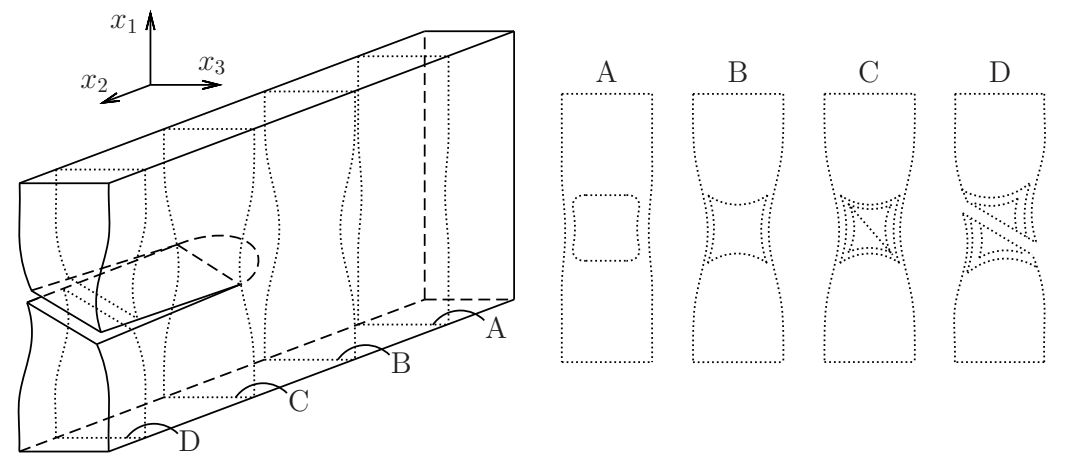

Figure 1: Sequential fracture process governing crack advance in a ductile metal plate subject to mode I loading, (A) onset of local thinning, (B) severe local thinning, (C) intensification of shear localization, and (D) slant failure.

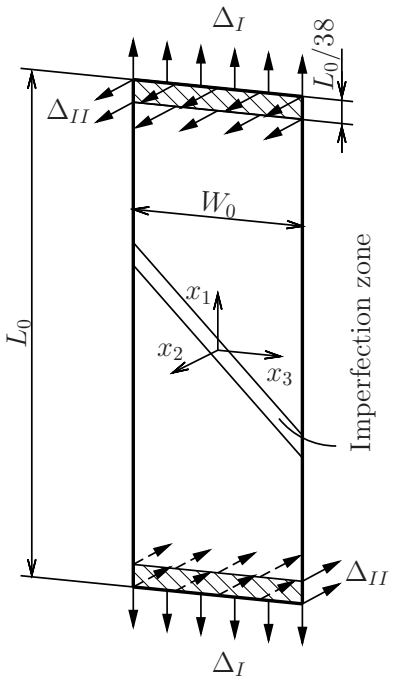

(a)

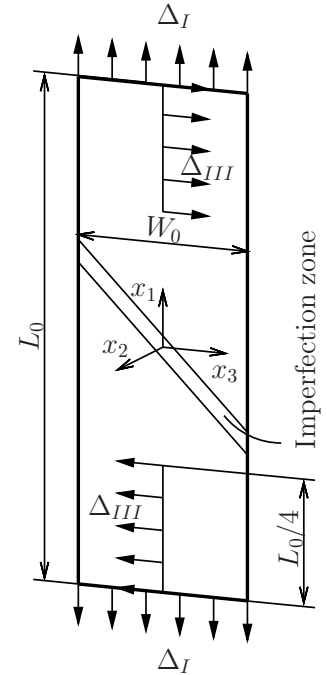

(b)

Figure 2: Illustrations of the two mode mixity models; (a) and (b) show the plate section with imperfection zone and imposed boundary conditions for the mode I-mode II and the mode I-mode III load case, respectively. Throughout the analyses, the initial ratio $L_{0} / W_{0}=3$ is kept constant. 


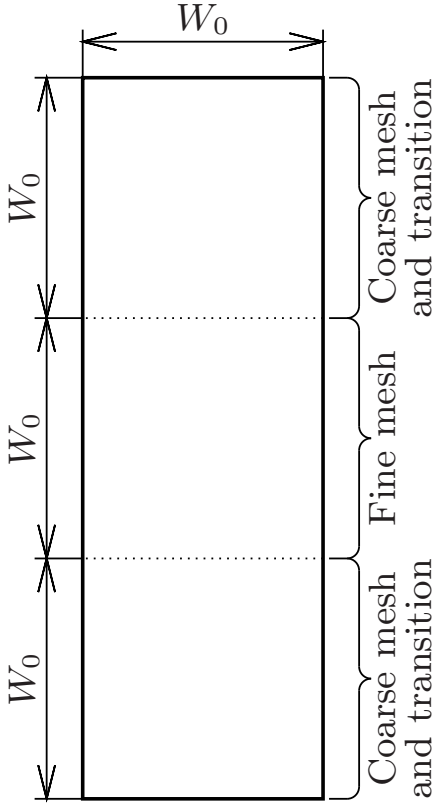

(a)

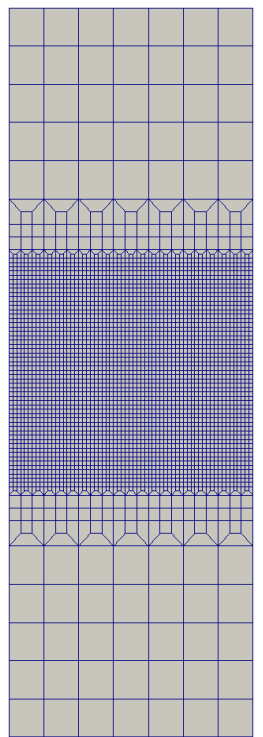

(b)

Figure 3: Discretization details; (a) shows the inner square section of the plate, where the fine mesh has been employed, together with the top and bottom regions, where a coarser mesh is adequate. (b) shows the discretized plate section considered in the present work where the side length of the elements is taken to be $L_{e}=W_{0} / 63$ in the fine region.

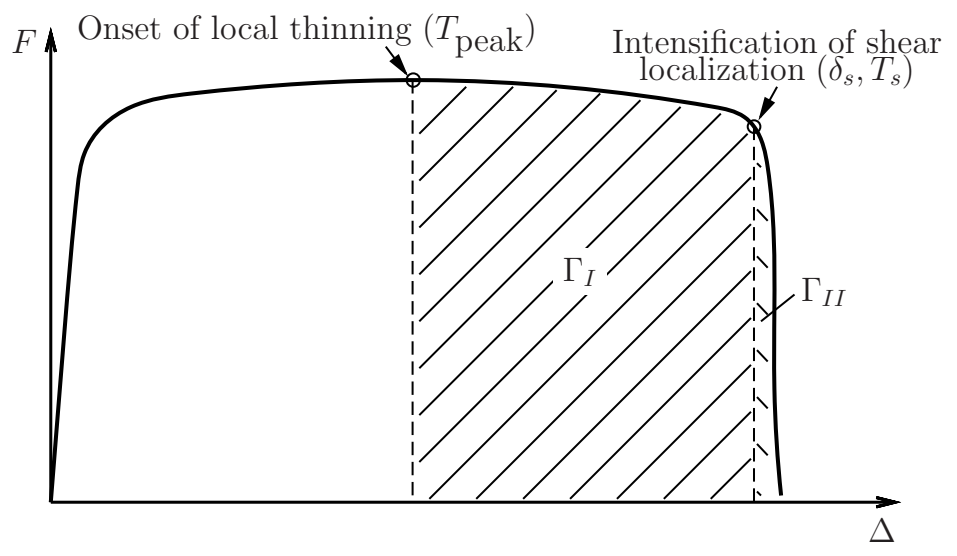

Figure 4: Illustration of the energy contributions, with $\Gamma_{I}$ being the cohesive energy contribution that governs the plate thinning process, and $\Gamma_{I I}$ being the energy contribution going into the subsequent shear localization and fracture. The cohesive energy is; $\Gamma_{0}=\Gamma_{I}+\Gamma_{I I}$. 


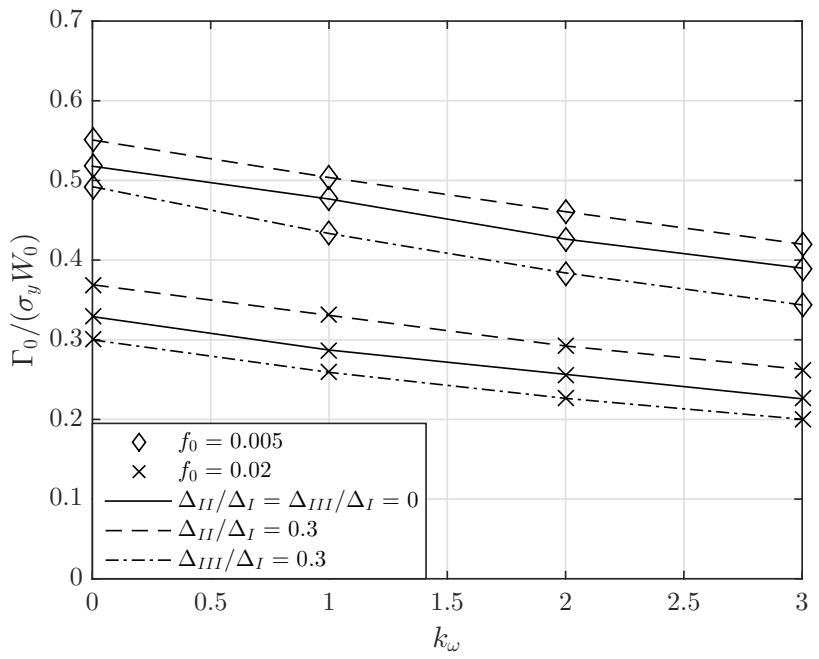

(a)

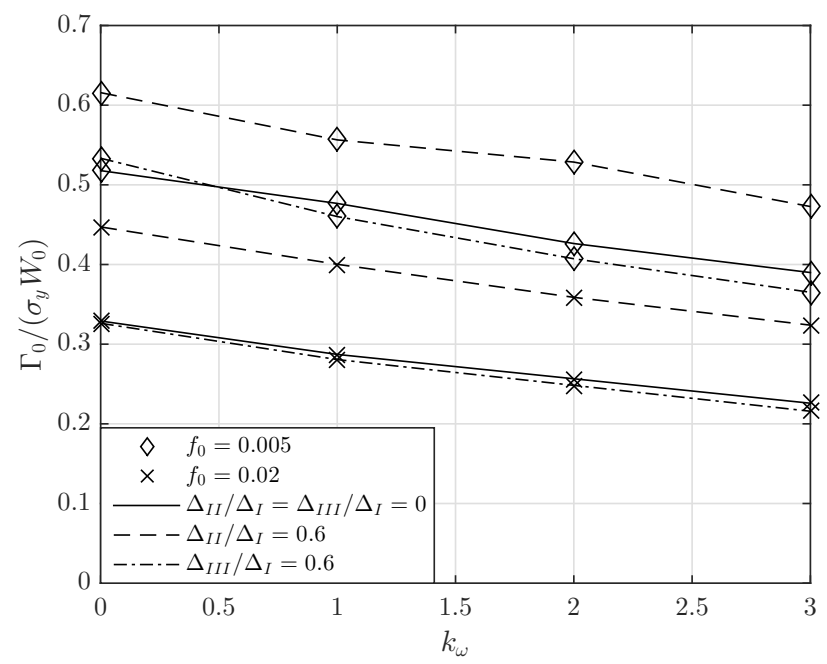

(b)

Figure 5: Development of the cohesive energy for various values of $k_{\omega}$ for pure mode I loading as well as for a) $\Delta_{I I} / \Delta_{I}=\Delta_{I I I} / \Delta_{I}=0.3$ and b) $\Delta_{I I} / \Delta_{I}=\Delta_{I I I} / \Delta_{I}=0.6$. Results are shown for two extreme values of the initial porosity, $f_{0}$. 


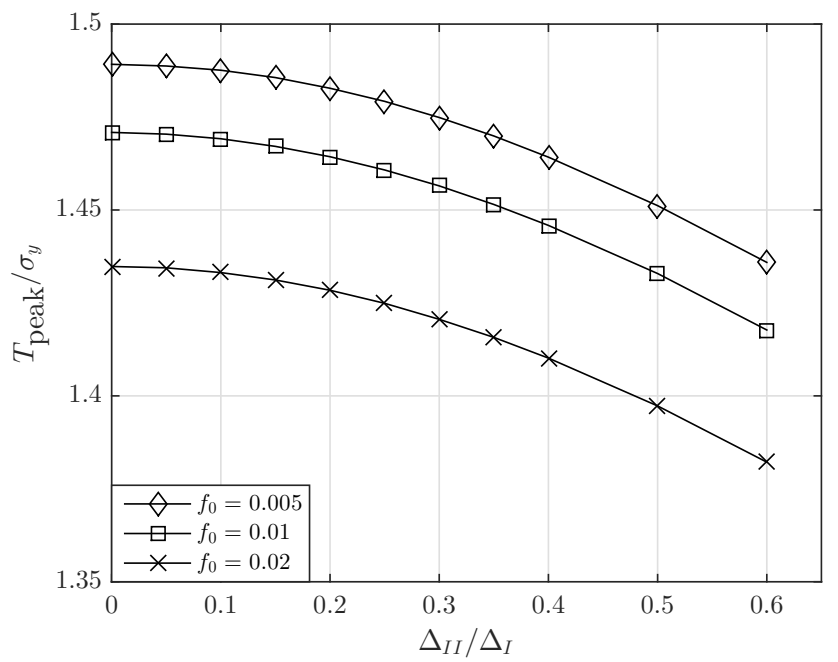

Figure 6: Development of the peak traction for various mode mixity ratios of $\Delta_{I I} / \Delta_{I}$. Results are shown for three values of initial porosity, $f_{0}$. 


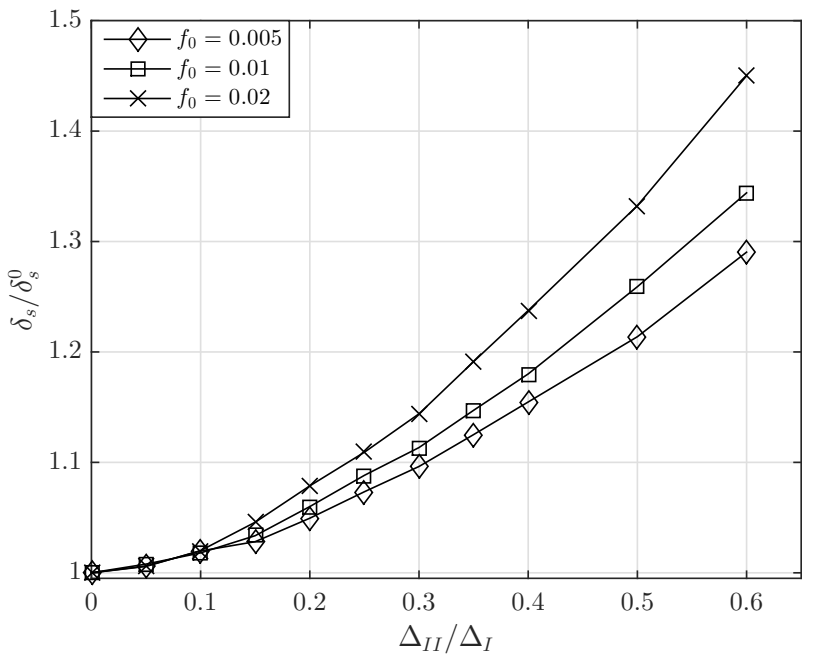

(a)

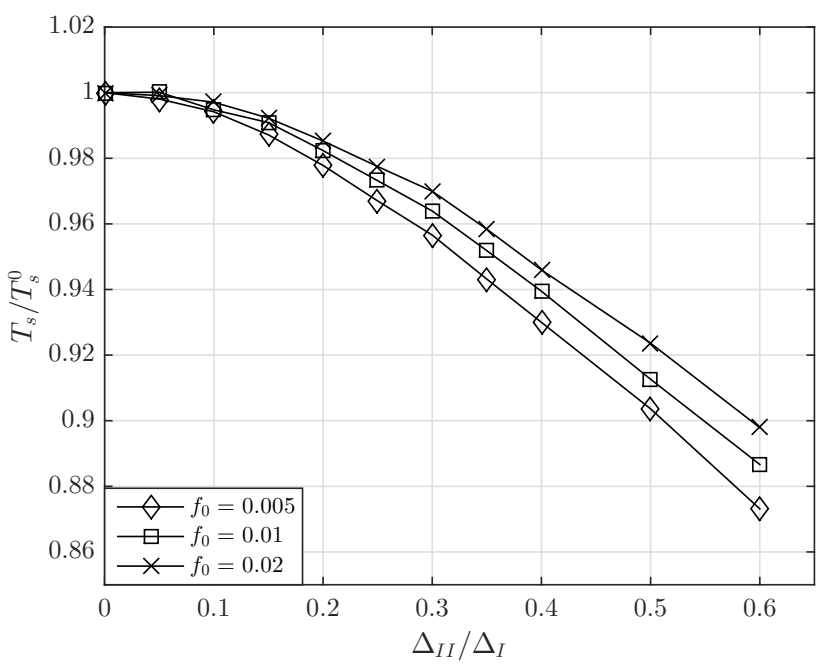

(b)

Figure 7: Development of the (a) displacement, $\delta_{s}$, and (b) traction, $T_{s}$, at intensification of the shear localization for various mode mixity ratios of $\Delta_{I I} / \Delta_{I}$ and for three values of initial porosity. The displacements and tractions are normalized with displacement and traction, respectively, for the pure mode I load case being $\delta_{s}^{0}=[0.1162 ; 0.0975 ; 0.0749]$ and $T_{s}^{0}=[1.2647 ; 1.2969 ; 1.3115]$ for $f_{0}=[0.005 ; 0.01 ; 0.02]$, respectively. 


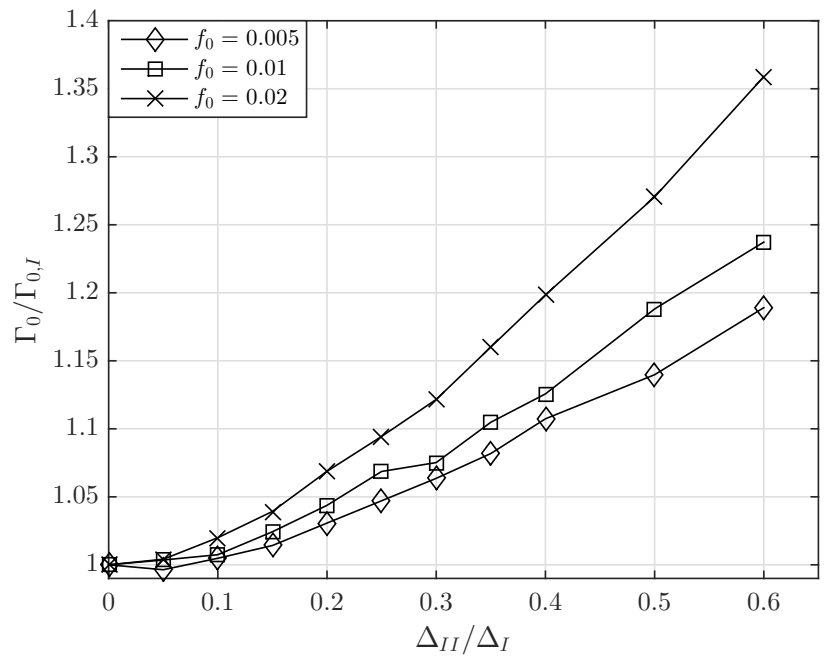

Figure 8: Development of the cohesive energy for various mode mixity ratios of $\Delta_{I I} / \Delta_{I}$ for three values of the initial porosity, $f_{0}$. The energies are normalized with the value for the pure mode I load case, in this case $\Gamma_{0, I} /\left(\sigma_{y} W_{0}\right)=[0.5178 ; 0.4339 ; 0.3291]$ for $f_{0}=[0.005 ; 0.01 ; 0.02]$, respectively. 


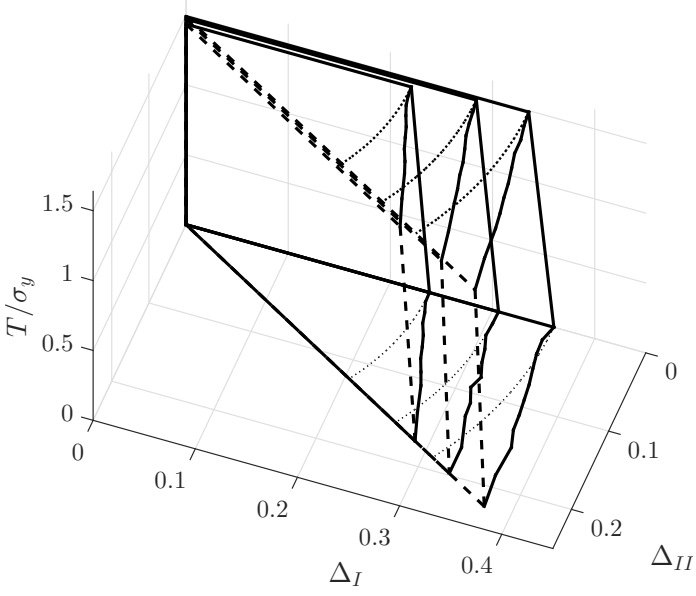

(a) View 1.

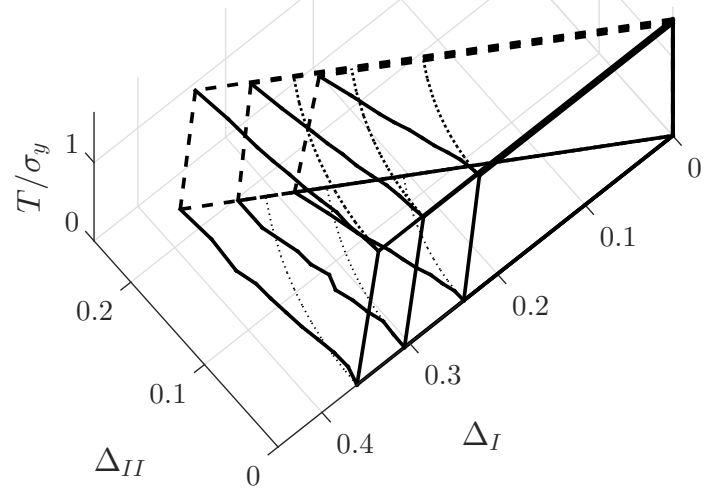

(b) View 2 .

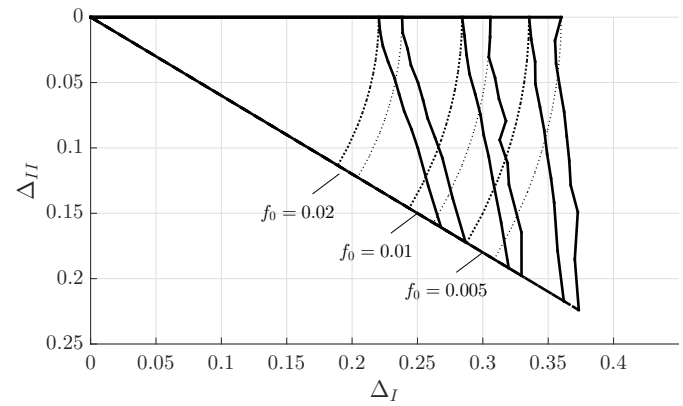

(c) View 3 .

Figure 9: Comparing the rotational sweep of the mode I curve with the micro-mechanics based mode mixity curves. Dotted lines are the sweep of the pure mode I bi-linear traction-separation relation whereas the bold lines are the corresponding bi-linear traction-separation relation fit to the results obtained in this study for a varying mode mixity ratio of $\Delta_{I I} / \Delta_{I}$, here shown for $f_{0}=$ $[0.005,0.01,0.02]$.

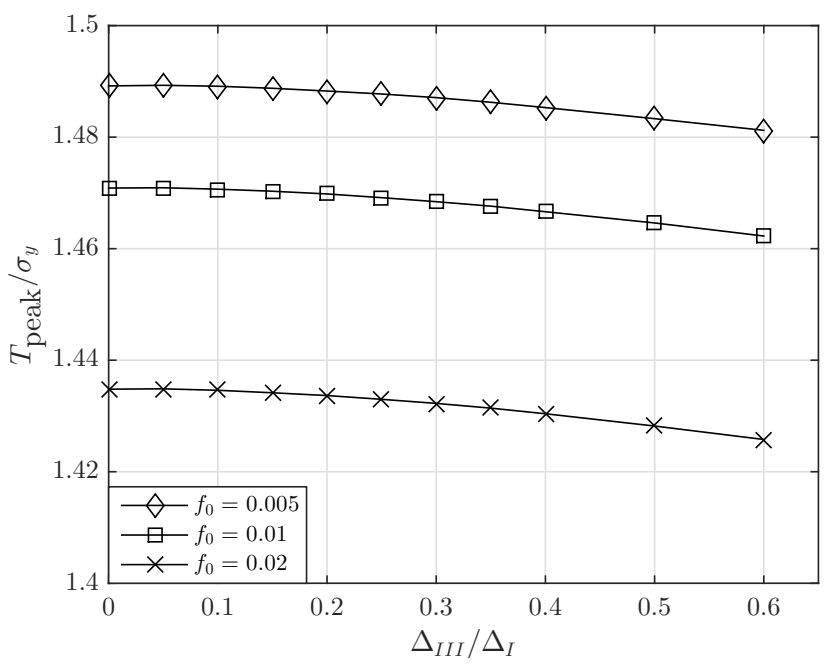

Figure 10: Development of the peak traction for various mode mixity ratios of $\Delta_{I I I} / \Delta_{I}$. Results are shown for three values of initial porosity, $f_{0}$. 


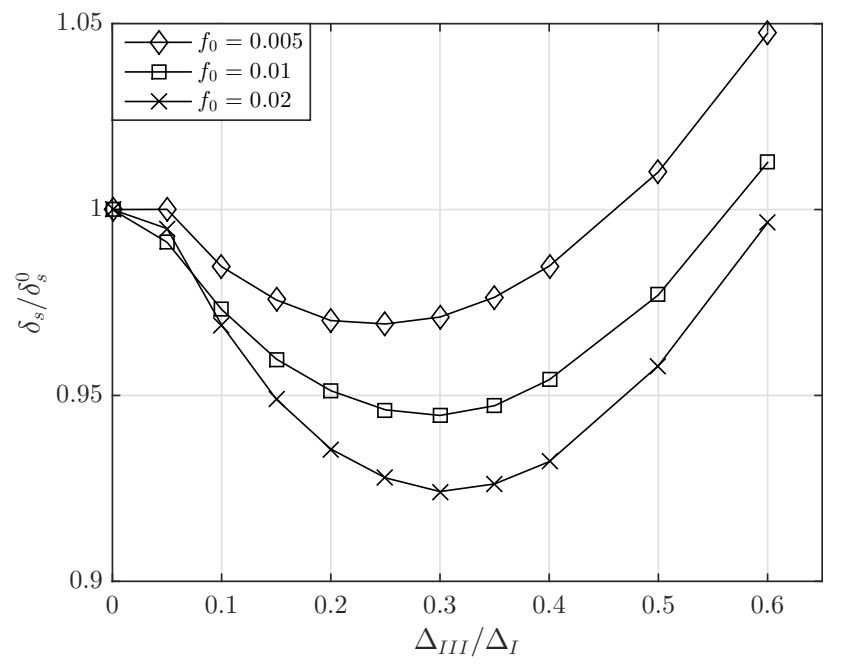

(a)

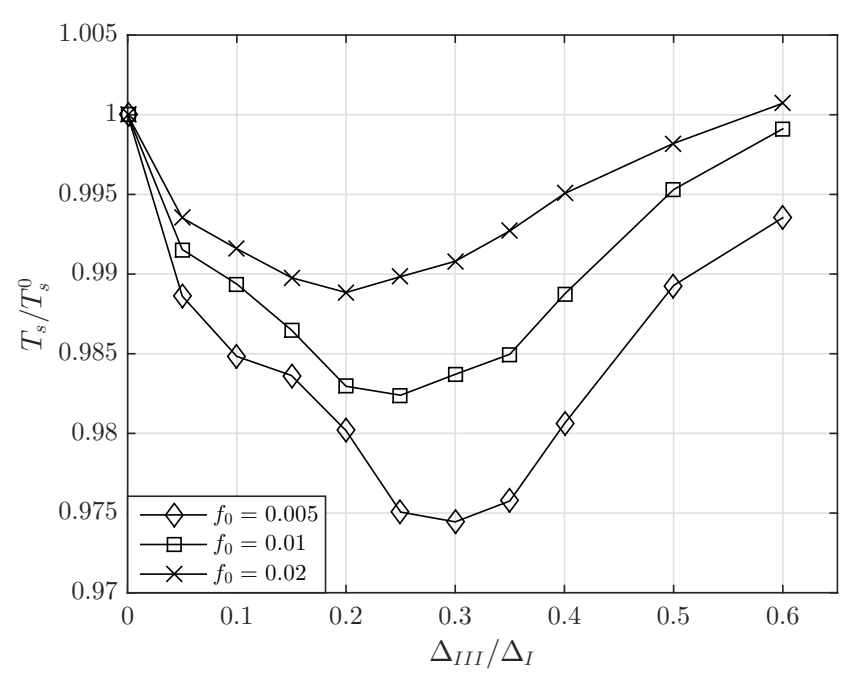

(b)

Figure 11: Development of the (a) displacement, $\delta_{s}$, and (b) traction, $T_{s}$, at intensification of the shear localization for various mode mixity ratios of $\Delta_{I I I} / \Delta_{I}$ and for three values of initial porosity. The displacements and tractions are normalized with displacement and traction, respectively, for the pure mode I load case being $\delta_{s}^{0}=[0.1162 ; 0.0975 ; 0.0749]$ and $T_{s}^{0}=[1.2647 ; 1.2969 ; 1.3115]$ for $f_{0}=[0.005 ; 0.01 ; 0.02]$, respectively. 


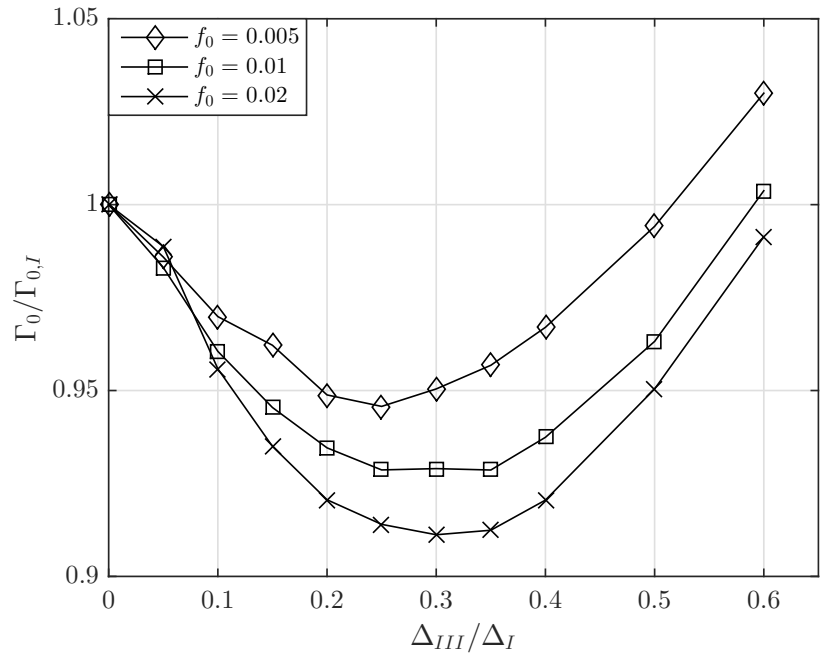

Figure 12: Development of the cohesive energy for various mode mixity ratios of $\Delta_{I I I} / \Delta_{I}$ for three values of the initial porosity, $f_{0}$. The energies are normalized with the value for the pure mode I load case, in this case $\Gamma_{0, I} /\left(\sigma_{y} W_{0}\right)=[0.5178 ; 0.4339 ; 0.3291]$ for $f_{0}=[0.005 ; 0.01 ; 0.02]$, respectively. 


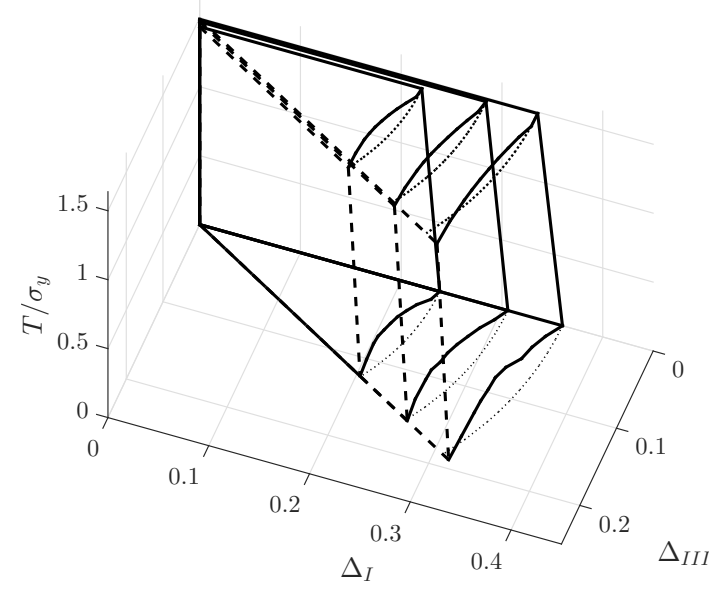

(a) View 1.

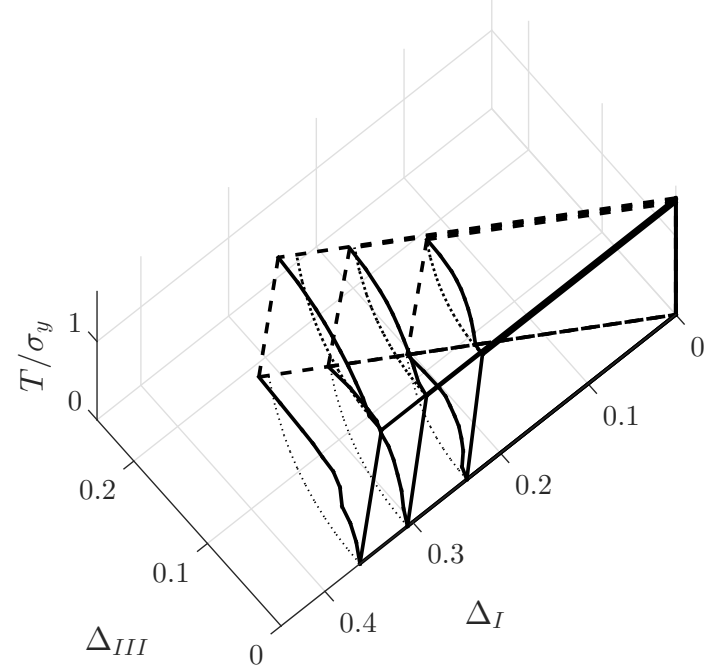

(b) View 2.

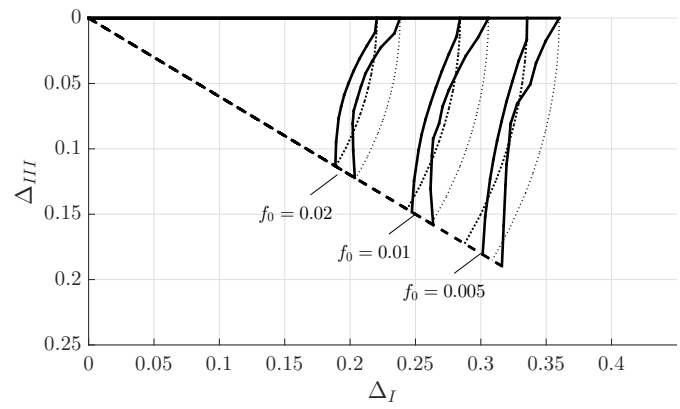

(c) View 3 .

Figure 13: Comparing the rotational sweep of the mode I curve with the micro-mechanics based mode mixity curves. Dotted lines are the sweep of the pure mode I bi-linear traction-separation relation whereas the bold lines are the corresponding bi-linear traction-separation relation fit to the results obtained in this study for a varying mode mixity ratio of $\Delta_{I I I} / \Delta_{I}$, here shown for $f_{0}=$ $[0.005,0.01,0.02]$. 


\section{Appendix A. Bi-linear cohesive relation}

Figure A.14 presents the bi-linear traction-separation relation chosen as reference in the present study. In this illustration, $T_{\text {peak }}$ is the peak traction, $\Gamma_{0}$ is the cohesive energy, and $\delta_{s}$ defines the separation at the intensification of the shear localization.

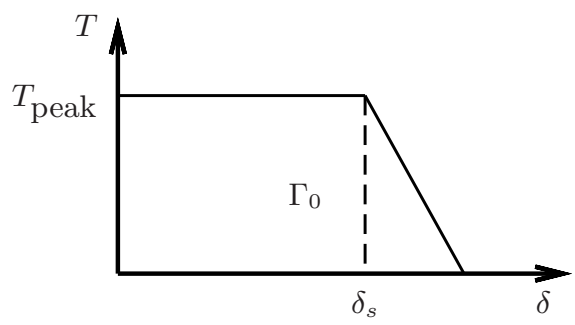

Figure A.14: The bi-linear traction-separation relation employed as basis for parameterizing the micromechanics based numerical results. 